\title{
DIÁLOGO Y APRENDIZAJE PERCIBIDO EN ESTUDIANTES DE MODALIDAD VIRTUAL: ABORDAJE CUALITATIVO EN UN PROGRAMA UNIVERSITARIO EN COSTA RICA
}

\section{(DIALOG AND PERCEIVED LEARNING IN ONLINE STUDENTS: A QUALITATIVE APPROACH IN A GRADUATE PROGRAM IN COSTA RICA)}

\author{
Edgar Salgado García \\ Universidad Latinoamericana de Ciencia y Tecnología (ULACIT), Costa Rica

\section{RESUMEN}

Se realizó un estudio exploratorio, de orden cualitativo, acerca de la percepción que tiene un grupo de 50 estudiantes de posgrado de una universidad costarricense, acerca de la intensidad del diálogo que se establece, en cursos virtuales, entre profesores y estudiantes, y entre los mismos estudiantes, en comparación con su experiencia en cursos presenciales. A partir del análisis, realizado mediante "software" para procesar datos cualitativos, se obtiene que ambos tipos de diálogo guardan relación con el nivel de aprendizaje percibido por los estudiantes en modalidad virtual. El papel del docente y la autonomía de los estudiantes son factores importantes que deben estudiarse con mayor profundidad.

Palabras clave: educación a distancia, educación virtual, cursos en línea, aulas virtuales, diálogo, Costa Rica.

\begin{abstract}
A qualitative and exploratory study was performed of the perceptions of a group of 50 graduate students from a Costa Rican university. The objective was to measure the intensity of online interactions between instructors and students, and between the students themselves. We then compared the results with traditional face-to-face (onsite) courses. Based on an analysis using qualitative data processing software, it was observed that there was a clear relationship with the perceived learning of online students for both dialogue types. The instructor's role, as well as student autonomy, were considered two important factors that should be studied in more detail.
\end{abstract}

Keywords: distance education, electronic learning, online courses, virtual classrooms, dialogue, Costa Rica.

Los principales modelos teóricos sobre la educación a distancia tienen como una de sus bases los conceptos de interacción y diálogo. La enseñanza y el aprendizaje son fenómenos eminentemente sociales; no puede ser de otro modo. Enseñar y aprender 
cobran sentido y relevancia en el diálogo. Todas las transacciones que tienen lugar en la educación, sea presencial o a distancia, deben establecer un diálogo, el cual puede, no obstante, estar diferido en el tiempo, atravesado por la distancia física entre actores, o estar mediado por el texto o por otras tecnologías (García Aretio, 2008). Sin embargo, al final el aprendizaje descansa en una comunicación multidireccional que sostiene el discurso y permite aprender a través de la constante realimentación.

Ante la llegada de la modalidad de enseñanza virtual a las universidades, como toda nueva forma de hacer las cosas, se generan dudas y expectativas por parte de todos los actores de los sistemas educativos: administradores, docentes, estudiantes, padres de familia, empleadores y el público en general. La idea de una educación superior en la que los estudiantes y los profesores no están presentes en un salón de clase, y pensar además en la otorgación de títulos académicos a personas que siguen programas de esta índole, causa algunas inquietudes, al menos en un principio. En países como los Estados Unidos, se ha llegado a determinar que existe una aceptable confianza por parte del público en los programas universitarios impartidos por medios virtuales (Lederman, 2013).

A pesar de haberse introducido en el país desde finales de los años noventa y principios de la década del dos mil (Hernández Pereira, 2005), la educación a distancia con medios virtuales ha sido poco estudiada en Costa Rica. Es un imperativo actual conocer los alcances de la utilización de las plataformas basadas en Internet para impartir cursos y programas académicos, sus fortalezas y debilidades, los mecanismos de adaptación de estudiantes y docentes a esta modalidad, así como su efectividad para promover aprendizajes significativos y que contribuyan verdaderamente al logro de los perfiles profesionales.

Evaluar el impacto de la educación virtual no es una tarea fácil, y sin duda involucra esfuerzos de medición, triangulación, seguimiento en el tiempo, acceso a distintas fuentes de información, etc. Y en el caso de la educación virtual, es muy difícil resistirse a compararla con los métodos de enseñanza que se han utilizado tradicionalmente en los centros educativos, sobre todo en las universidades.

Comparar la educación a distancia con la educación presencial es una tarea compleja, y a la vez polémica. Diversos estudios, que han analizado cientos de investigaciones sobre efectividad de la educación a distancia a través de la técnica de meta-análisis, han llegado a la conclusión de que los resultados de aprendizaje en educación a distancia no difieren significativamente de los obtenidos mediante la enseñanza presencial (Russell, 1999; Smith, Jaggars y Bailey, 2010).

Ahora bien, los estudios sobre "efectividad" del aprendizaje en ambas modalidades han recurrido típicamente a mediciones "objetivas", como desempeño en pruebas estandarizadas, exámenes de preguntas cerradas, calificaciones obtenidas o tasas de 
aprobación. Pero no conocemos mucho acerca de la "experiencia" de los estudiantes desde su propia mirada ${ }^{1}$.

En nuestros países latinoamericanos, y en particular en Costa Rica, no abundan las investigaciones sobre cómo experimentan los estudiantes su proceso de aprendizaje en la modalidad virtual, y si las interacciones que allí tienen lugar están o no contribuyendo al desarrollo de sus conocimientos y habilidades. Por ello cobra relevancia plantear un estudio exploratorio, que utilice un método cualitativo y que permita trazar algunos puntos de partida para más adelante profundizar y ampliar las perspectivas sobre el fenómeno de la educación "online”.

De acuerdo con lo anterior, las preguntas a las que el presente estudio intentó dar respuesta fueron:

- ¿Cuál es la noción que tienen los estudiantes acerca del concepto de "diálogo"?

- ¿Cómo consideran los estudiantes que tiene lugar el diálogo entre el docente y los alumnos en los cursos virtuales, en comparación con los presenciales?

- ¿Cómo consideran los estudiantes que tiene lugar el diálogo entre compañeros en los cursos virtuales, en comparación con los presenciales?

- ¿Cuál es el grado de aprendizaje percibido por parte de los estudiantes en cursos virtuales, en comparación con los presenciales?

- ¿Cuáles factores, situaciones de contexto o características de los diálogos que se entablan con los docentes y entre los alumnos se relacionan con un mayor o menor aprendizaje percibido por parte de los estudiantes?

\section{Diálogo}

Existen tres grandes vertientes en el estudio del diálogo que interesa aquí destacar. Primero, la filosófica, siendo uno de sus máximos exponentes el austriacoisraelí Martin Buber (Arroyo Arrayás, 2007). Segundo, la tradición rusa, en la que se encuentran el lingüista Mijail Bakhtin y el pedagogo Lev Vigotsky. Es a través de este último que se enfatiza el papel de la construcción social de los conocimientos, proceso que tiene como base el diálogo. Y en tercer lugar, en Latinoamérica, la obra del educador brasileño Paulo Freire.

El diálogo implica un intercambio entre dos o más personas, por medio del lenguaje o algún sistema simbólico, en el que las partes se escuchan, se respetan, se alternan, proponen y expresan sus ideas, puntos de vista y sentimientos, sin ser juzgados; el diálogo incluye además la puesta en común, la valoración y el común acuerdo. 
En el ámbito educativo, el diálogo es el elemento central a través del cual se establece la relación entre docentes y estudiantes, y por medio del cual se aprende en comunidad. En la educación a distancia, particularmente, encontramos el concepto de "conversación didáctica guiada", de Holmberg, así como la más reciente propuesta de Lorenzo García Aretio, el "diálogo didáctico mediado". Ambos dan cuenta del factor "distancia" al utilizar los calificativos de "guiado" (Holmberg) o "mediado" (García Aretio), dando a entender que el diálogo se entabla a partir de un medio o canal de comunicación, como el texto o los medios tecnológicos.

La mayoría, si no todos, los estudiantes y docentes, coincidirán en que el diálogo es un elemento fundamental para aprender y enseñar. El diálogo puede ser inmediato, o diferido, o entre individuos que están presentes, o a través de algún medio de comunicación; puede ser "real”, o incluso "simulado" (García Aretio, 2008).

\section{Distancia}

La distancia es el elemento que caracteriza a una modalidad educativa en la que no hay coincidencia espacio-temporal entre los participantes. De alguna manera, la distancia se convierte en la barrera por vencer, a través de los medios tecnológicos y las diversas estrategias de enseñanza propias de este tipo de enseñanza. El papel que juega el concepto de distancia está plasmado en uno de los principales modelos conceptuales de este tipo de enseñanza: La teoría de distancia transaccional de Michael Moore (1993). Tres factores interactúan en este modelo: diálogo, estructura y autonomía. Cada uno de ellos varía en función de los demás. Así, por ejemplo, a menor diálogo, se requiere una mayor autonomía por parte del estudiante; a mayor estructura, el aprendizaje puede darse con una menor intensidad de diálogo; ante una menor autonomía del estudiante, se necesita de un grado mayor de estructura, etc.

Otro de los modelos, quizás el más importante, que se ha desarrollado para caracterizar las interacciones que tienen lugar en la educación a distancia, enfocado en el aprendizaje virtual u "online", es el de "comunidad de indagación" "community of inquiry") (Garrison, Anderson y Archer, 2000). En este, la experiencia educativa de los estudiantes está determinada por la confluencia de tres factores: la presencia pedagógica, la presencia cognitiva y la presencia social.

La presencia pedagógica se relaciona con el papel que juega el docente como instructor y facilitador del discurso; la presencia cognitiva, con las interacciones entre los estudiantes y el contenido del curso o programa formativo; y la presencia social, con el establecimiento de un clima propicio para la interacción entre los mismos estudiantes, lo cual brinda un sentido de pertenencia y de conexión entre los integrantes del grupo. La "comunidad de indagación" se sostiene a partir de estos tres pilares, o "presencias". 


\section{Pesos y contrapesos}

Se ha estudiado la forma como los componentes de los modelos de distancia transaccional y comunidad de indagación pueden compensar la disminución de intensidad en alguno de ellos, o incluso su ausencia. Encontramos así cursos o programas a distancia en los que se supone que la presencia social podría mitigar la ausencia del docente, o en los que la falta de contenidos estructurados puede ser compensada por un mayor grado de interacción con el docente, etc.

Estas interacciones son bastante complejas, y no siempre puede establecerse una relación inequívoca entre los factores que intervienen en los modelos conceptuales. Existe una multiplicidad de factores del contexto social, del entorno tecnológico, de los modelos de enseñanza, del apoyo institucional, e incluso factores internos al individuo, como estilos de aprendizaje, actitudes, motivaciones o estilos cognitivos, que entran en juego en la experiencia de aprendizaje.

Algunos autores han determinado los factores asociados a una mayor satisfacción y a un mayor nivel de aprendizaje percibido, como los contenidos de los cursos y el diseño de los mismos (Barberá, Clará y Linder-Vanberschot, 2013). En otros estudios se ha encontrado que el grado de involucramiento del docente (presencia pedagógica) es determinante, es decir, es el factor que se relaciona con mayor fuerza con la satisfacción y el aprendizaje percibido de los estudiantes (véase, al respecto, la discusión planteada por Garrison, 2007).

\section{MÉTODO}

\section{Tipo de estudio}

Se eligió un enfoque cualitativo, debido al carácter exploratorio de la presente investigación. El método se inspiró en el enfoque general inductivo propuesto por Thomas (2006). Tal como señala este autor, "el análisis inductivo se refiere a los enfoques que utilizan principalmente lecturas detalladas de datos crudos para derivar conceptos, temáticas o un modelo a través de interpretaciones hechas a partir de los datos por parte de un evaluador o investigador" (p. 238). Es decir, se pretende alcanzar unos objetivos, o en nuestro caso, responder a unas preguntas predeterminadas, pero a la vez establecer relaciones entre los datos que permitan construir un modelo conceptual que pueda dar cuenta del fenómeno abordado. 


\section{Contexto institucional}

El estudio se llevó a cabo en la Universidad Latinoamericana de Ciencia y Tecnología (ULACIT), ubicada en la ciudad de San José, Costa Rica, una universidad privada, con una población de 2600 alumnos y 250 profesores. La universidad imparte carreras de grado y posgrado en las áreas de Ingenierías, Ciencias Sociales, Salud y Ciencias Empresariales. Desde hace más de diez años ofrece programas de posgrado completamente virtuales y combina cursos de esta modalidad con las lecciones presenciales.

La institución se adhiere a un modelo pedagógico socioconstructivista, basado en el marco de la Enseñanza para la Comprensión (EpC) (Gardner y Mansilla, 1994; Stone-Wiske, 1998; Perkins y Blythe, 1994). Desde este enfoque, no se administran exámenes tradicionales y se incentiva la participación de los estudiantes, el trabajo colaborativo y la elaboración de proyectos aplicados, denominados "desempeños de comprensión", como forma de evaluar las competencias adquiridas. Una reciente investigación permitió determinar que existe una buena aceptación del modelo pedagógico por parte de los estudiantes y egresados (Salgado, 2012). Los docentes reciben capacitación en estrategias de enseñanza virtual para el nivel universitario, y se cuenta con el sistema de gestión de aprendizaje Blackboard Learn Enterprise, versión 9, como plataforma para el desarrollo de los cursos.

\section{Participantes}

En el estudio participaron 50 estudiantes de los programas de posgrado de la Facultad de Ciencias Empresariales. Los estudiantes pertenecían a los programas de Maestría en Administración de Empresas (MBA), con distintos énfasis, así como a la Maestría en Gerencia de Proyectos. Estas maestrías ofrecen cursos tanto presenciales como virtuales.

De conformidad con el enfoque cualitativo, el grupo de participantes se seleccionó a partir de ciertos criterios de interés. De este modo, se elaboró una lista de todos los estudiantes matriculados en el primer trimestre de 2014, que tuviesen un avance mínimo del 50\% de su carrera, y que hubiesen cursado al menos un $75 \%$ de las asignaturas en modalidad virtual. A partir de estos criterios, se obtuvo un total de 172 estudiantes. A todos ellos se les envió una invitación a participar en el estudio por medio del correo electrónico, dando como resultado el grupo de 50 alumnos que formó parte de la investigación. Es de notar que la decisión de responder el cuestionario enviado no estuvo condicionada de manera alguna, ni formó parte de una evaluación formal de los cursos o los docentes por parte de la universidad. 


\section{Instrumentos}

Se diseñó una guía de cuatro preguntas abiertas, a saber:

- ¿Cómo definiría, en sus propias palabras, el concepto de «diálogo»?

- Desde su propia experiencia como estudiante en cursos y programas presenciales (en los que asiste regularmente a un salón de clase con un profesor y otros compañeros) y en cursos virtuales (en los que se utiliza una plataforma informática para comunicarse a través de la Internet), ¿̇ha percibido alguna diferencia en cuanto a la calidad del diálogo que se establece entre profesores y alumnos? Cuéntenos sobre qué diferencias ha encontrado, o si cree que no las hay, justifique su respuesta.

- En cuanto al diálogo que se establece entre los mismos estudiantes (entre compañeros), ¿nota alguna diferencia en su experiencia como alumno(a) de cursos virtuales en comparación con los cursos presenciales? Cuéntenos en qué consisten esas diferencias, si las ha notado, o si cree que no las hay, explique por qué.

- Partiendo de su experiencia, ¿qué tanto percibe que ha aprendido en la modalidad virtual en comparación con la presencial? Por favor indique si nota diferencias en su nivel de aprendizaje entre las modalidades, y si cree que las hay, cuáles son los factores que para usted explican esa diferencia.

El cuestionario fue enviado a los casilleros electrónicos personales de cada estudiante por medio de la plataforma de administración de encuestas en línea "Survey Monkey"2. El mensaje incluyó una explicación del propósito de la investigación, indicaciones acerca del carácter voluntario de la participación, así como los datos de contacto del investigador para evacuar cualquier duda.

\section{Análisis de los datos}

Se procedió a una lectura completa de todas las respuestas dadas por los participantes, y a partir de ellas, se elaboró una lista de códigos. Cada respuesta se clasificó dependiendo de si la opinión expresaba un nivel de diálogo mayor, igual o menor en los cursos virtuales en comparación con los cursos presenciales. Asimismo, se asignaron códigos a los factores explicativos, como "el profesor no retroalimenta", "los compañeros aportan al debate desde su experiencia profesional", o "el grado de aprovechamiento depende del estudiante", etc.

Los códigos se ajustaron, uniendo algunos de ellos que parecían repetitivos o que podían condensarse en uno solo, y atendiendo también al criterio de "saturación" propio de la metodología de investigación cualitativa. Este proceso dio como 
resultado un conjunto de 10 a 15 códigos por pregunta, los cuales se categorizaron según temáticas comunes.

El análisis de la información recogida se llevó a cabo con los siguientes recursos:

El programa "Atlas Ti"3, un reconocido paquete de "software" para efectuar análisis de datos cualitativos, por medio de la asignación de códigos, la construcción de categorías y el establecimiento de relaciones entre ellas. Con la ayuda de esta herramienta, se procedió a generar y analizar tablas de coincidencias de códigos, con el fin de identificar los factores asociados con la intensidad del diálogo y el aprendizaje percibido.

La aplicación en línea "Wordle" , la cual genera "nubes de palabras" con base en la frecuencia con que aparecen las palabras en un texto.

Con la ayuda de estas herramientas, y con el apoyo adicional de Microsoft Excel, se hizo un análisis de los casos en que los estudiantes reportaron mayores, iguales o menores grados de diálogo y de aprendizaje percibido, con el fin de establecer relaciones entre los textos codificados, utilizando para ello categorías más abstractas que agruparan varios códigos. Esto permitió identificar las principales relaciones y tendencias de los datos, para llegar finalmente a sistematizarlos.

\section{RESULTADOS Y DISCUSIÓN}

Los resultados y su consiguiente discusión se presentan seguidamente como respuesta a las seis interrogantes planteadas en la introducción. Debido a la naturaleza cualitativa de la investigación, no se ha prestado atención a frecuencias absolutas o relativas u otros indicadores cuantitativos, sino a los temas emergentes $\mathrm{y}$ a las relaciones entre ellos.

\section{¿Cuál es la noción que tienen los estudiantes acerca del concepto de "diálogo"?}

En general, los estudiantes relacionaron el diálogo con conceptos tales como intercambio, comunicación o conversación entre dos o más personas, en la cual se ponen en común ideas, opiniones e información sobre temas específicos. Se contempló la interacción tanto en el plano verbal (oral) como escrito. El análisis de frecuencia de términos, realizado mediante "Wordle", dio como resultado la "nube de palabras" que se muestra en la figura 1. 


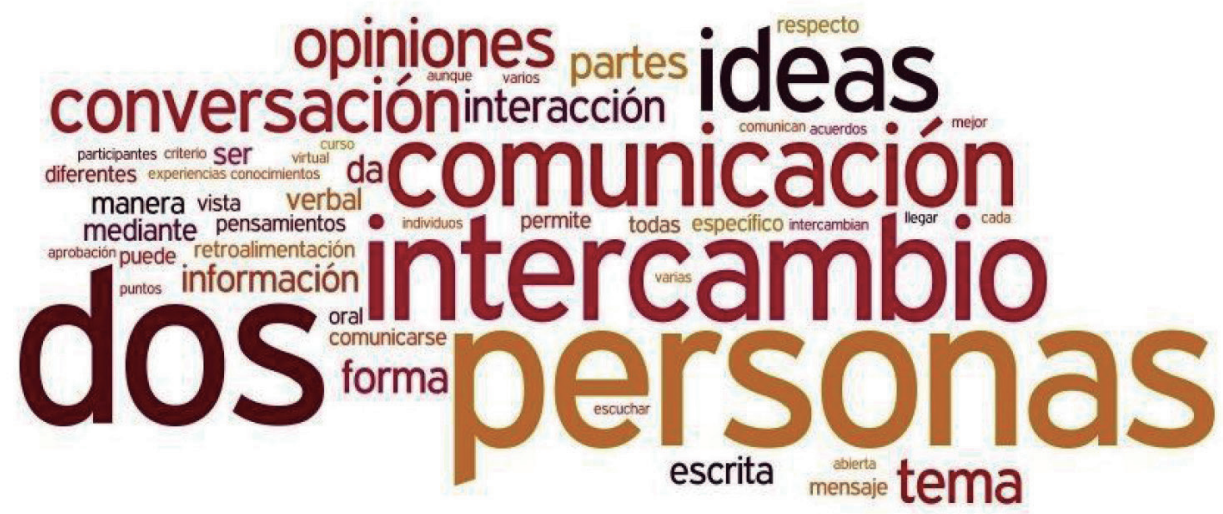

Figura 1. "Nube de palabras", generada por la herramienta "Wordle", de las respuestas dadas por los estudiantes ante la pregunta “¿cómo definiría, en sus propias palabras, el concepto de "diálogo"?

No se encontró alguna diferencia importante entre el concepto de diálogo que expresaron los alumnos y sus percepciones sobre el grado en que este se refleja en los cursos virtuales. Sin embargo, esta primera pregunta cumplió la función de establecer el concepto central alrededor del cual girarían las siguientes preguntas. Asimismo, permitió tener claridad acerca de qué entienden los estudiantes por "diálogo", concepto que en general no varió mucho entre las respuestas dadas por ellos. Llamó la atención, eso sí, que muy pocos estudiantes se refirieran al diálogo como un proceso para la búsqueda de acuerdos comunes. Lo que es claro es que para el grupo de estudiantes, "diálogo" es un intercambio que se da entre personas, que puede ser verbal o escrito, primordialmente.

\section{¿Cómo consideran los estudiantes que tiene lugar el diálogo entre el docente y los alumnos en los cursos virtuales, en comparación con los presenciales?}

Las principales categorías que emergieron del análisis se relacionan con la inmediatez del diálogo en la enseñanza presencial frente a su carácter diferido en la modalidad virtual. Quienes consideraron que el diálogo con el profesor es de menor intensidad en los cursos virtuales, opinaron en torno a una menor frecuencia y calidad de la retroalimentación ofrecida por el docente, así como una relación disminuida en términos de empatía, cercanía, y de posibilidades de atender a las emociones o el lenguaje no verbal (véase tabla 1).

Los estudiantes que manifestaron que existe un diálogo igual o mayor en cursos virtuales, recurrieron a argumentos relacionados con el papel determinante que 
juega el profesor como interlocutor, su nivel de participación y la rapidez con que obtienen respuesta por parte de él.

\section{Tabla 1.}

Categorías relacionadas con la percepción de la intensidad del diálogo entre el profesor y los estudiantes en cursos virtuales, en comparación con cursos presenciales

\section{Menor diálogo con el profesor}

- El diálogo con el profesor es más lento, el profesor tarda más en responder.

- El profesor no retroalimenta.

- Menor empatía, cercanía, relación; no se aprecian emociones, gestos, lenguaje no verbal.

- Depende del tipo de curso.

\section{Igual diálogo con el profesor}

- El papel del profesor es determinante.

- El profesor sí responde, muestra un buen nivel de involucramiento.

- Las herramientas virtuales pueden compensar la no presencia física del profesor.

- Depende del estudiante.

- Depende del tipo de curso.

\section{Mayor diálogo con el profesor}

- El papel del profesor es determinante.

- Las herramientas virtuales pueden compensar la no presencia física del profesor.

Seguidamente, en la tabla 2, se presentan cuatro temas generales que pudieron extraerse de las respuestas de los estudiantes ante la pregunta referida al nivel de diálogo que se establece con el profesor. 


\section{Tabla 2.}

Diálogo entre profesor y estudiantes. Temas centrales y ejemplos representativos de citas textuales de las respuestas de los estudiantes

1. El papel del profesor es determinante. Cuando no interviene, no responde preguntas o tarda mucho en hacerlo, se percibe una menor intensidad de diálogo en la modalidad virtual

1.1. Solo los profesores buenos dan retroalimentación y comentan sobre los trabajos de los estudiantes en los foros y sección de anuncios. En otros de los cursos, la interacción es muy poca, por lo que realmente no hay diálogo.

1.2. De manera presencial se hacen diálogos directos e inmediatos y se dan desde mi punto de vista en mayor cantidad, a diferencia que de manera virtual que se hacen de manera retardada y en ciertos cursos virtuales son escasos de parte del profesor.

1.3. La principal diferencia es que la interacción no se da al mismo tiempo. Se puede entrar a un foro e ingresar una opinión, pero la respuesta no se recibe al instante. Es un proceso más lento.

1.4. Mientras que en clases presenciales, hay retroalimentación, en cursos virtuales, muchos profesores establecen comunicación de manera unidireccional, ellos brindan las guías para realizar trabajos y la retroalimentación es nula o muy pobre.

2. Al no tener al profesor presente físicamente, no se puede apreciar el lenguaje no verbal o sus gestos; no hay empatía, cercanía o conexión

2.1. Considero que el diálogo es más enriquecedor en clases presenciales porque las expresiones no verbales aportan un valor a la comunicación que no se transmite fácilmente por Internet, creo que una mirada o incluso el timbre de voz del profesor causa un impacto en los estudiantes.

2.2. Las relaciones interpersonales se ven seriamente afectadas en el sentido de que no existe esa parte humana, cara a cara, que puede manejarse dentro de una clase presencial. Por tal motivo, la capacidad de diálogo presencial no es el mismo desde la perspectiva del diálogo virtual, ya que se carece de la capacidad de entablar una visión cara a cara y eso es fundamental en el diálogo.

2.3. El diálogo a mi criterio es más efectivo cuando se realiza presencialmente y no con un intermediario (informático en este caso) que puede corregir faltas ortográficas pero no puede sensibilizar las palabras. 
3. Un uso adecuado por parte del docente de las herramientas que provee el campus virtual puede compensar la falta de cercanía

3.1. En un curso presencial el diálogo tiende a ser más disperso, en la que cuenta la personalidad de los alumnos y el profesor, mientras que en un curso virtual, existe mayor oportunidad de reflexión. Tuve el caso de un profesor que hacía teleconferencias todos los domingos con nosotros, a manera de tutorial. La interacción personal puede llegar hasta donde el profesor lo decida.

3.2. En cuanto a la calidad del diálogo sigue siendo la misma, esto gracias a la comunicación por chat, teléfono e inclusive video llamadas.

3.3. Dependiendo del profesor puede ser inclusive mejor, ya que las personas al interactuar en un foro, wiki o reunión virtual están más preparadas o estudiadas con el tema.

4. El contacto con el docente dependen también del tipo de asignatura y de la motivación e interés de los estudiantes

4.1. Por lo tanto aunque ambos el virtual y el presencial es enriquecedor, desde mi perspectiva es adecuado la utilización de ambos dependiendo del tema a tratar. Es mi opinión que la educación implique presencia y virtual, más en un tema como es la Gerencia de Proyectos y donde el trabajo es interactuar con personas.

4.2. Siento que al ser virtual, queda bajo responsabilidad de cada quien la calidad con la que se desempeñe en el curso, la experiencia es obviamente diferente, pero es mejor virtual pues permite hacer las clases dentro de un rango de tiempo menos limitado.

4.3. No hay mucha diferencia entre cursos presenciales y virtuales el estudiante con sus ganas y méritos intenta obtener los conocimientos.

\section{¿Cómo consideran los estudiantes que tiene lugar el diálogo entre compañeros en los cursos virtuales, en comparación con los presenciales?}

Las percepciones sobre el nivel de diálogo que se establece entre los compañeros de grupo en cursos virtuales giran alrededor de temas como el menor sentido de pertenencia, la empatía disminuida (al no haber cercanía física), la poca participación de los compañeros, así como la responsabilidad que deben asumir los estudiantes al intervenir en los cursos (véase tabla 3). Un tema importante que surgió fueron las ventajas de la asincronía propia de los cursos a distancia: tener más tiempo para elaborar las aportaciones y la desinhibición de algunos estudiantes que muestran timidez para participar en cursos presenciales. 
Tabla 3.

Categorías relacionadas con la percepción de la intensidad del diálogo entre compañeros en cursos virtuales, en comparación con cursos presenciales

\section{Menor diálogo entre compañeros}

- Al no estar frente a frente hay menor debate, interacción; es más difícil coordinar los trabajos.

- Los compañeros no participan, no aportan, lo hacen por obligación.

- Menor sentido de pertenencia, empatía, cercanía; no se aprecia el lenguaje no verbal ni las emociones.

\section{Igual diálogo entre compañeros}

- Buena coordinación entre los compañeros al realizar los trabajos.

- Depende de la responsabilidad del estudiante.

- Depende del papel facilitador del profesor.

- Los compañeros que son tímidos en clases presenciales participan más en las discusiones virtuales.

- Un buen uso de las herramientas virtuales pueden compensar la ausencia física de los compañeros.

- Mayor tiempo para preparar, reflexionar y fundamentar los aportes.

\section{Mayor diálogo entre compañeros}

- Los compañeros aportan desde sus experiencias profesionales.

- Los compañeros que son tímidos en clases presenciales participan más en las discusiones virtuales.

- Mayor tiempo para preparar, reflexionar y fundamentar los aportes.

En cuanto a los temas centrales, elaborados a partir del análisis de categorías, destacan los que se presentan en la tabla 4. Es de resaltar la importancia de factores como lenguaje no verbal, sentido de pertenencia y empatía, que aparecen como centrales en el rubro del diálogo entre pares. Asimismo, los estudiantes se refirieron a una variedad de factores condicionantes que entran en juego, como la motivación, el sentido de responsabilidad, e incluso el papel del docente como facilitador y diseñador de actividades interactivas. 
Tabla 4.

Diálogo entre compañeros de grupo. Temas centrales y ejemplos representativos de citas textuales de las respuestas de los estudiantes

1. Al no poder conocer a los compañeros presencialmente, no se puede percibir el lenguaje no verbal ni sus emociones; hay un menor sentido de pertenencia, empatía y relación

1.1. Al carecer el entorno de la interacción personal, es evidente que la interacción y el diálogo se ven disminuidos.

1.2. No se evidencian las expresiones verbales, las cuales son el $90 \%$ de la comunicación real, esto en cursos virtuales a diferencia de cursos presenciales.

1.3. En clases presenciales uno puede conocer a sus compañeros e identificar con cuáles podría uno sentirse más cómodo trabajando en los proyectos con el fin de lograr los mejores resultados, esto es casi imposible cuando a uno le toca un grupo virtual con compañeros de los que no se tiene referencias y entonces queda de cierta forma al azar el resultado del trabajo grupal.

1.4. Algunas veces los diálogos se vuelven directos con palabras como "no estoy de acuerdo", "no mencionó.....", "No considero...”, esta situación al no poseer un tono humano se puede terminar mal interpretando y genera sentimientos encontrados.

2. Algunos estudiantes no participan con frecuencia, no aportan a las discusiones, y otros lo hacen solo por obligación

2.1. A pesar de que como estudiante se puede investigar más y analizar a profundidad, la comunicación entre estudiantes es poca.

2.2. No he notado mayor diferencia salvo la que puedan hacer los mismos compañeros que no participan mucho o sus comentarios no fomentan una discusión provechosa.

2.3. Me parece que los cursos virtuales son una buena plataforma para poder estudiar cuando uno pasa muy enredado o incluso debe ausentarse de la ciudad o del país, sin embargo el espacio para la interacción y comunicación se limita al grupo de trabajo o los foros de discusión.

2.4. En los cursos virtuales se hace para salir del paso de una tarea, en un curso presencial el diálogo se convierte en debate, por lo cual la interacción es más rica en contenido y puntos de vista. 
3. Hay muchos "dependes": la calidad del diálogo con los compañeros depende de la motivación y la responsabilidad de los mismos estudiantes; del papel del profesor; del uso que se haga de las herramientas tecnológicas, y puede depender también del tipo de curso

3.1. Personalmente no he tenido ningún problema, es cuestión del compromiso del estudiante y su responsabilidad.

3.2. Depende mucho de que el trabajo que asignó el profesor permita trabajar a distancia (como creo que debería ser).

3.3. Puede ser complicado en un inicio pero después de ser contactados para un trabajo grupal nos pasamos los contactos de Skype, Whatsapp o correo electrónico.

3.4. Depende del curso, en algunas ocasiones el diálogo fluye como si se estuviera en clase.

\section{¿Cuáles es el grado de aprendizaje percibido por parte de los estudiantes en cursos virtuales, en comparación con los presenciales?}

Los estudiantes que consideraron que existe un menor grado de aprendizaje en cursos virtuales fundamentaron su respuesta en razones como la lentitud del diálogo, la escasa retroalimentación por parte del docente, el no tener oportunidad de conocer a sus compañeros cara a cara, y el no tener el profesor a su disposición para hacerle preguntas en el momento, entre otras (véase tabla 5).

Por otra parte, quienes afirmaron haber logrado un aprendizaje igual o mayor en la modalidad virtual destacaron, principalmente, la mayor autonomía que debe ejercer el alumno. Entre los que expresaron haber aprendido más en cursos virtuales, aparecen también categorías relacionadas con el mayor tiempo para preparar sus participaciones, la flexibilidad y el ahorro en transporte.

\section{Tabla 5 .}

Categorías relacionadas con el grado de aprendizaje percibido en cursos virtuales, en comparación con cursos presenciales

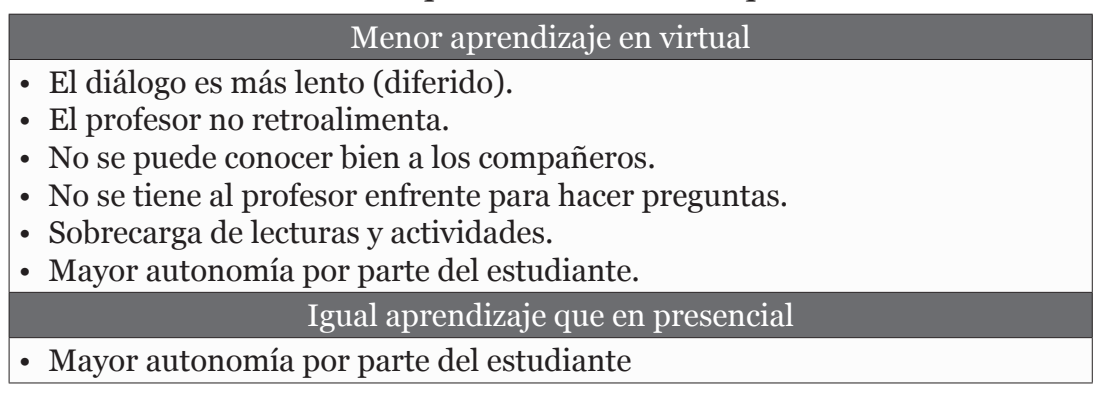




\section{Mayor aprendizaje en virtual}

- Mayor autonomía del estudiante.

- Más tiempo para pensar lo que se va a decir.

- Es más flexible, más personalizado.

- Sobrecarga de lecturas y actividades.

- Se ahorra en transporte, alimentación, etc.

En la tabla 6 se pueden apreciar citas textuales de las respuestas de los estudiantes, que fueron agrupadas en tres grandes temas sobre el grado de aprendizaje percibido. Es de notar que estos temas apuntan a la autonomía y al papel del docente, y dentro de este último, la función de diseño de las actividades de forma que propicien la interacción con este y entre los mismos estudiantes.

Tabla 6.

Aprendizaje percibido por parte de los estudiantes. Temas centrales y ejemplos representativos de citas textuales de las respuestas de los estudiantes

1. El ejercicio de una mayor autonomía por parte del estudiante se relaciona con la percepción de un mayor aprendizaje en cursos virtuales

1.1. Personalmente considero que en cursos virtuales podemos aprender más que en cursos presenciales ya que debemos asimilar la información no con la explicación presencial de un profesor, sino más bien con la retroalimentación virtual que él nos brinde, además de efectuar más lecturas, análisis, e investigaciones que en cursos presenciales. Por ejemplo, sino tenemos claro algún tema o concepto indagamos más que cuando le pedimos la explicación al profesor en clase.

1.2. Los cursos virtuales exigen mucha investigación sobre los temas, la guía de los profesores es menor por lo que requiere mucho mayor esfuerzo de los estudiantes, me encanta esta modalidad virtual permite maximizar el tiempo en todo sentido.

1.3. Las ventajas de la modalidad virtual es la necesidad de leer mucho y aprender investigando. Caso contrario a la presencial en que las personas esperan que les sirvan todo en bandeja.

1.4. La modalidad virtual por si misma obliga a leer más, a investigar más y en general a trabajar mucho más.

1.5. Creo que el nivel de exigencia personal es mayor. 
2. Los elementos motivacionales (dedicación, perseverancia, interés) para aprender efectivamente en un curso virtual

2.1. Yo considero que uno aprende en la modalidad virtual tanto como uno lo desea. Es decisión de cada persona si quiere realizar todas las lecturas y trabajos a consciencia.

2.2. En realidad los temas son los mismos, la cuestión es que en la modalidad virtual hay que adaptarse y poner mucho más interés en sacarle provecho a dichos temas, es un dilema personal, cada quien verá si quiere sacarle el mayor provecho posible a cada curso ya sea este virtual o presencial.

3. El aprendizaje depende en gran medida de la habilidad del profesor para diseñar actividades adecuadas el entorno virtual

3.1. De nuevo quiero enfatizar en que la modalidad de participación del profesor con sus alumnos es esencial. En algunos casos la documentación y forma de organizarse del profesor hace que exigidamente se tengan que leer los contenidos del curso, de forma que la aclaración de dudas se puedan evacuar con el profesor o compañeros de curso.

3.2. La claridad en la que ponen los temas, ejercicios y proyectos del profesor, esto se logra con una comunicación efectiva estudiante-profesor y viceversa.

3.3. En realidad he aprendido mucho en ambas, sin embargo, considero que el nivel de aprendizaje adquirido no difiere de lo virtual o presencial sino más del profesor, metodología, programa del curso y desarrollo. Ya que, he tenido excelente aprendizaje virtual como presencial, y no tan satisfactorio en ambas modalidades por igual dependiendo del curso.

¿Cuáles factores, situaciones de contexto o características de los diálogos que se entablan con los docentes y entre los alumnos se relacionan con un mayor o menor aprendizaje percibido por parte de los estudiantes?

Finalmente, sellevó a cabo un análisis delas respuestas dadas por cada participante en el estudio, a partir del cual se elaboró la tabla 7, que sintetiza la relación entre las intensidades de diálogo y el aprendizaje percibido de los estudiantes. Téngase en cuenta que al hablar de "mayor", "igual" o "menor", se está siempre en el contexto de la comparación entre la modalidad virtual y la presencial. 
Tabla 7.

Relación entre las intensidades del diálogo con el profesor y entre compañeros, y el grado de aprendizaje percibido en cursos virtuales, en comparación con los presenciales

\begin{tabular}{|l|l|c|c|c|}
\cline { 3 - 5 } \multicolumn{2}{c|}{} & \multicolumn{4}{c|}{$\begin{array}{c}\text { Aprendizaje percibido } \\
\text { Menor }\end{array}$} & Igual & Mayor \\
\hline \multirow{2}{*}{$\begin{array}{l}\text { Diálogo con el } \\
\text { profesor }\end{array}$} & Menor & $\mathrm{x}$ & & \\
\hline & Igual & & $\mathrm{x}$ & $\mathrm{x}$ \\
\cline { 2 - 6 } $\begin{array}{l}\text { Diálogo con los } \\
\text { compañeros }\end{array}$ & Mayor & & & \\
\cline { 2 - 6 } & Igual & & $\mathrm{x}$ & $\mathrm{x}$ \\
\cline { 2 - 6 } & Mayor & & & \\
\hline
\end{tabular}

Como se observa en la tabla 7, un menor aprendizaje percibido en cursos virtuales estuvo siempre relacionado con una apreciación de menor diálogo con el profesor y entre los compañeros. De manera similar, la percepción de un nivel de aprendizaje igual entre cursos virtuales y presenciales se relacionó más con una percepción de igual nivel de diálogo con el docente y con los pares. El grupo más variable fue el de quienes consideraron que su nivel de aprendizaje ha sido mayor en cursos virtuales. Entre ellos, la mayoría consideraron que el grado de interacción con los profesores y compañeros fue igual al de cursos presenciales, e incluso algunos consideraron que uno u otro fue menor. Al hacer un análisis detallado de las citas textuales codificadas, se logró determinar que el elemento que "inclina la balanza", es decir, que tiene un peso importante en la percepción de un mayor grado de aprendizaje en la modalidad virtual, tiene que ver con la autonomía del estudiante y su grado de responsabilidad. Los estudiantes que así opinaron tendieron a percibir, además, esta mayor autonomía y responsabilidad, como características deseables de la educación virtual, y que les mueven a continuar aprendiendo. También se encontró que la autonomía tiende a asociarse con una mayor satisfacción de los estudiantes con los cursos virtuales (véanse nuevamente las citas 1.1 a 1.5, y 2.1 a 2.2, de la tabla 6). 


\section{CONCLUSIONES}

Se ha realizado un primer acercamiento al fenómeno de cómo experimentan los estudiantes de posgrado de una universidad costarricense el diálogo con sus profesores y con sus compañeros, y su relación con el grado de aprendizaje percibido en cursos virtuales, en comparación con su experiencia en cursos presenciales.

El abordaje cualitativo permitió asomarse a un fenómeno muy poco estudiado en el contexto de este país, pudiéndose así trazar algunas líneas para futuras investigaciones que puedan explorar, con mayor detalle, estos y otros factores relacionados con la experiencia educativa en entornos virtuales.

Los resultados tienden a apoyar el modelo de distancia transaccional de Moore, en el sentido de que una menor intensidad de diálogo puede compensarse mediante una mayor dosis de autonomía por parte de los estudiantes. Asimismo, desde el modelo de "comunidad de indagación", pudo notarse que si bien la presencia social juega un papel importante en la promoción del aprendizaje, el papel del docente ocupa un lugar difícilmente sustituible. El papel del profesor fue un tema recurrente incluso en su función propiciadora de interacción entre los mismos estudiantes, a partir del diseño y de la conducción de las actividades.

Es posible que las diferencias encontradas entre los grados de aprendizaje percibido se deban a factores muy diversos, pero que a partir de esta investigación pueden empezar a dilucidarse, a través de nuevos estudios. Al parecer, un mayor grado de aprendizaje percibido en cursos virtuales requiere al menos de una intensidad de diálogo equivalente entre la modalidad virtual y la presencial, desde la perspectiva de los estudiantes. Sería conveniente investigar más acerca de las características de los estudiantes que valoran la autonomía como un elemento positivo y deseable en los cursos virtuales, en contraposición a otros estilos de relación más dependientes de la figura del profesor. El papel de este último se revela como primordial, pero las funciones que cumple pueden variar en función de las preferencias, estilos de aprendizaje y expectativas de los estudiantes. 


\section{NOTAS}

1. No se trata de determinar cuál de las dos modalidades, la presencial o la virtual, es más o menos "efectiva". Se intenta, más bien, conocer cómo los estudiantes perciben las interacciones dialógicas con el profesor y con sus pares, las cuales constituyen el "núcleo" o centro de la experiencia de aprendizaje.

2. Se utilizó la versión Profesional, con licencia. Para mayor información sobre Survey Monkey y sus especificaciones, puede verse: www.surveymonkey.com

3. Se utilizó la versión 7. Atlas Ti es producido por Atlas.ti GmbH, Berlín, Alemania. Para mayor información sobre sus características y aplicaciones, puede verse: www.atlasti. $\underline{\text { com }}$

4. Este es un recurso gratuito, disponible en: www.wordle.net

\section{REFERENCIAS BIBLIOGRÁFICAS}

Arroyo Arrayás, L. M. (2007). La antropología dialógica en la historia de la filosofía. Themata, Revista de Filosofía, 39, 301307.

Barberá, M., Clará, M., y Linder-Vanberschot, J. A. (2013). Factors influencing student satisfaction and perceived learning in online courses. E-learning and Digital Media, 10(3), 226-335.

García Aretio, L. (2008). Diálogo didáctico mediado. Editorial del Boletín Electrónico de Educación a Distancia (BENED), junio. Madrid, España: Cátedra UNESCO de Educación a Distancia.

Gardner, H., y Mansilla, V. (1994). Teaching for understanding in the disciplines-and beyond. Teachers College Record, 96(2), 198-218.

Garrison, D. R. (2007). Online community of inquiry review: Social, cognitive, and teaching presence issues. Journal of Asynchronous Learning Networks, 11(1), 61-72.

Garrison, D. R., Anderson, T., y Archer, W. (2000). Critical inquiry in a text-based environment: Computer conferencing in higher education. The Internet and Higher Education, 2(2-3), 87-105.

Hernández Pereira, R. (2005). Estado del arte: Educación virtual en la educación superior en Costa Rica. En M. Otoya, y
L. Vargas (Eds.), Aprendizaje virtual y desarrollo sostenible: El rol de las universidades (pp. 29-40). Heredia, Costa Rica: Consorcio ELAC de la Unión Europea y Editorial Universidad Nacional (UNA).

Lederman, D. (2013). American adults see online courses as at least equivalent in most ways. Inside Higher Ed, 15 de octubre. Recuperado de http://www. insidehighered.com/news/2013/10/15/ american-adults-see-online-coursesleast-equivalent-most-ways

Moore, M. G. (1993). Theory of transactional distance. En D. Keegan (Ed.), Theoretical principles of distance education (pp. 2238). Londres, Inglaterra: Routledge.

Perkins, D. N., y Blythe, T. (1994). Putting understanding up front. Educational Leadership, 51(2), 4-7.

Russell, T. L. (1999). The no significant difference phenomenon. Raleigh, North Carolina, EE.UU: North Carolina State University Press.

Salgado, E. (2012). Enseñanza para la comprensión en la educación superior: la experiencia de una universidad costarricense. Revista Iberoamericana de Educación Superior, III(8), 34-50.

Smith Jaggars, S., y Bailey, T. (2010). Effectiveness of fully online courses for college students: Response to 
a Department of Education metaanalysis. New York: Community College Research Center, Teachers College, Columbia University. Recuperado de: http://ccrc.tc.columbia.edu/ publications/effectiveness-fully-onlinecourses.html

Stone-Wiske, M. (1998). Teaching for understanding: Linking research with practice. San Francisco, California: Jossey-Bass.
Thomas, D. R. (2006). A general inductive approach for analyzing qualitative evaluation data. American Journal of Evaluation, 27(2), 237-246.

Zhao, Y., Lei, J., Yan, B., Lai, C., y Hueyshan, S.T. (2005). What makes the difference? A practical analysis of research on the effectiveness of distance education. Teachers College Record, 107(8),18361884 .

\section{PERFIL ACADÉMICO Y PROFESIONAL DEL AUTOR}

Edgar Salgado García. Bachiller en Ciencias en Psicología, Florida State University. Licenciado en Psicología, Universidad de Costa Rica. Master en Ciencias, Southern Illinois University. Doctorando en Ciencias de la Educación, Universidad Católica de Costa Rica. Actualmente es Asesor de Investigación y Evaluación Institucional de ULACIT, Costa Rica.

E-mail: esalgado@ulacit.ac.cr

DIRECCIÓN DEL AUTOR

ULACIT

Apartado postal 10235, San José, 1000 Costa Rica

Fechas de recepción del artículo: 30/05/2014

Fecha de aceptación del artículo: 03/09/2014

\section{Como citar este artículo:}

Salgado García, E. (2015). Diálogo y aprendizaje percibido en estudiantes de modalidad virtual: abordaje cualitativo en un programa universitario en Costa Rica. RIED. Revista Iberoamericana de educación a Distancia, volumen 18, $\mathrm{n}^{\circ} 1$, pp. 191211. 